\title{
The ATLAS experiment educational printables
}

\author{
Mariana Velho ${ }^{a, *}$ on behalf of the ATLAS Collaboration \\ ${ }^{a}$ European Laboratory for Particle Physics, CERN, \\ Espl. des Particules 1, Meyrin, Switzerland \\ E-mail: mariana.velho@cern.ch
}

Five years after launching the first ATLAS Colouring Book, the ATLAS experiment increased the number of educational printables. We present here two ATLAS Colouring Books, the ATLAS Fact Sheets, the ATLAS Physics Cheat Sheets, and ATLAS Activity Sheets. These materials are intended to cover key topics of the work done by the ATLAS Collaboration and the physics behind the experiment for a broad audience of all ages and levels of experience. In addition, there is ongoing work in translating these documents to different languages, with one of the colouring books already available in 18 languages. These printables are prepared to complement the information found in all ATLAS digital channels, they are particularly useful in outreach events and in classroom activities.

The European Physical Society Conference on High Energy Physics (EPS_HEP2021)

26-30 July 2021

Online conference, jointly organized by Universität Hamburg and the research center DESY 


\section{Introduction}

Science is crucial to technological, social, cultural, and economic growth and it has a strong influence on all aspects of our daily life. Therefore, it is important that the whole universe of science is not confined to the space in which it is produced. Communication is the key to achieve that purpose. Communication is the link between the production of scientific knowledge and the general public [1]. This is particularly true for the large, publicly-funded international collaborations of the Large Hadron Collider (LHC) at CERN [2]. Not only does the public have the right to know how and towards what aim their resources are being spent, but they are key stakeholders of the knowledge that is being gained. The ATLAS Collaboration takes advantage of its communication platforms to bring these messages to a variety of global audiences, thus securing support for the field through key stakeholders and decision makers and sparking interest in the broader public and the next generation of scientists and engineers.

Relying on this premise that science must reach the public, a wide range of educational printables were developed communicating the research by the ATLAS experiment [3]. Audiences of all ages and levels of expertise can benefit from these visual resources to learn something about particle physics. The development of these printables was done believing that information is often better understood when presented in a clear, concise, and visually appealing way. Due to the recent pandemic situation, these educational printables also turned out to be very useful since they are easy to print - available in colour and black and white versions - and don't require any further equipment to teach particle physics concepts working both in a classroom setting but also individually at home.

\section{The ATLAS experiment colouring books}

\subsection{The ATLAS colouring book}

The first ATLAS Colouring Book [4] was launched in 2016, it is aimed at an audience between six and ten years old and for now is available in 18 languages. This book teaches about:

- The ATLAS detector, for example how it has different layers that are designed to detect different types of particles.

- Introduces the Standard Model and the concept that it is a "recipe" with the basic ingredients (elementary particles and forces) that make up everything.

- Highlights the international nature of the ATLAS experiment.
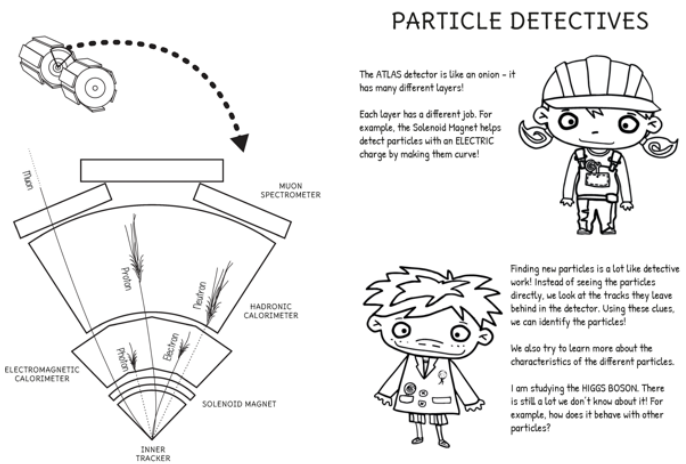

Figure 1: ATLAS detector slice in the ATLAS Colouring Book explaining the different layers of the ATLAS detector.

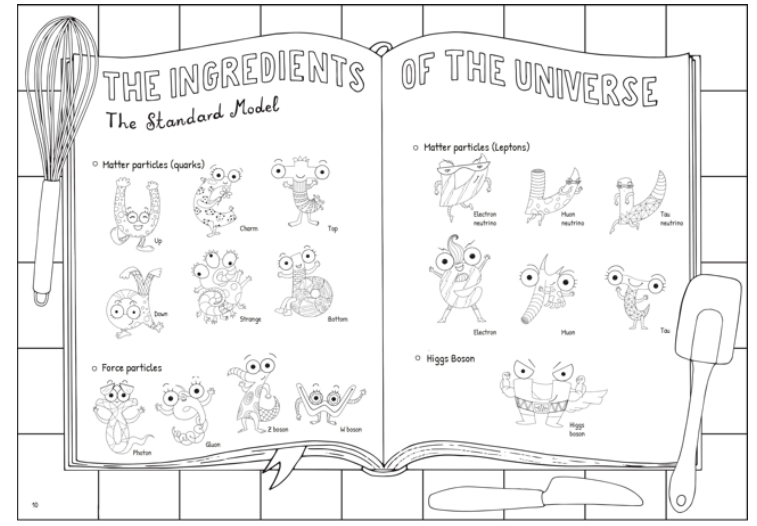

Figure 2: Standard Model represented as a recipe in which the ingredients are the elementary particles. 


\subsection{Particles of the Universe}

The 'Particles of the Universe' Colouring Book [5] is the second ATLAS colouring book and was launched in January 2021. This second colouring book builds upon the introduction of the Standard Model made in the previous colouring book and presents all the elementary particles in more detail. The elementary particles introduce themselves and reveal interesting facts about the role they play in the Universe. It also introduces the concept of scale where the path from atom to nucleus, proton/neutron to quarks is made and the concept of colour charge is introduced. This colouring book is for now available in ten languages.

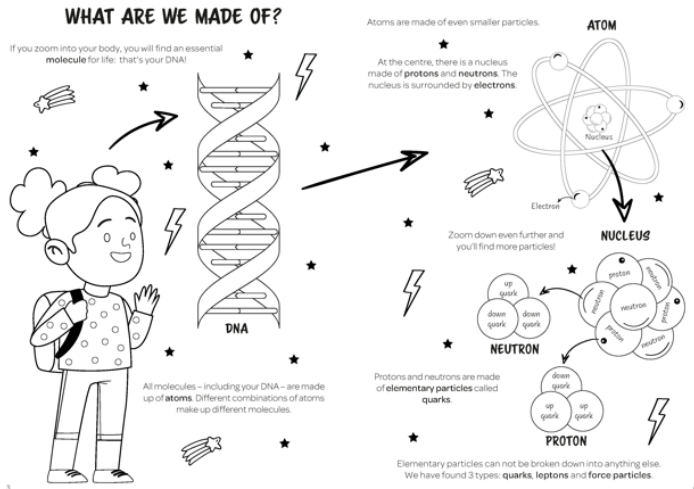

Figure 3: Introducing the scale concept and how elementary particles can not be broken down into anything else.

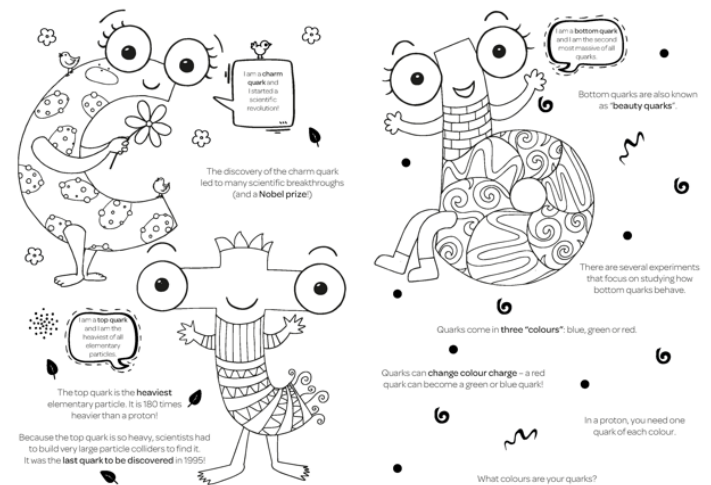

Figure 4: Elementary particles presenting themselves and interesting facts about them.

\section{The ATLAS sheets}

\subsection{The ATLAS Cheat Sheets}

The ATLAS Cheat Sheets [6] were developed with the goal to make the material on ATLAS public website [7] - such as briefings, news pieces - as well as ATLAS publications easier to comprehend and accessible to a wider audience. These sheets also provide resources for students and teachers (students beginning in particle physics and looking for definitions of common concepts all in one place, or professors looking for posters or handouts for classrooms). The target audience of these sheets is the general public interested in particle physics rather than experts, so using jargon or equations was avoided during their development. There are three sheets available as of now: 'Conservation Laws', 'Feynman Diagrams' and 'The Standard Model'.

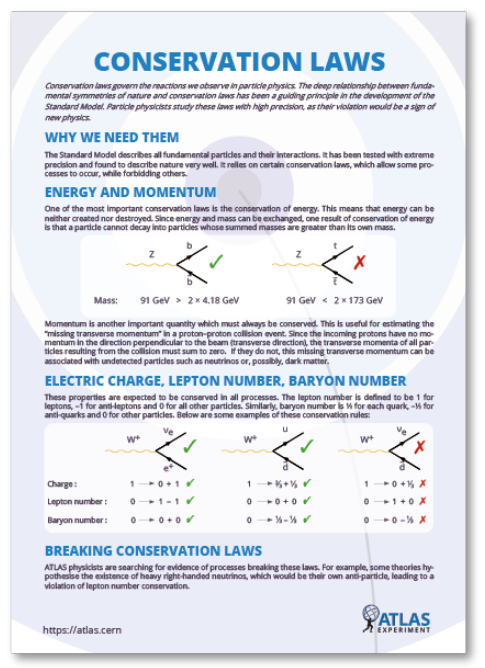

Figure 5: Example of an ATLAS Cheat Sheet about Conservation Laws. 


\subsection{The ATLAS Fact Sheets}

The ATLAS Fact Sheets [8] were developed with the aim of collecting facts about the ATLAS detector and the collaboration all in one place (for example: detector components; organisation of the ATLAS Collaboration; technology transfer) and to provide resources for students, teachers, and guides (students looking for clear, up-to-date facts about ATLAS; posters or handouts in classrooms). The target audience of these sheets are people like the ATLAS guides but also the general public that is interested in ATLAS and wants to learn some more details (they are the more detailed of all the ATLAS educational printables, but still designed to be understood by the general public). There are ten fact sheets available in three languages as of now.

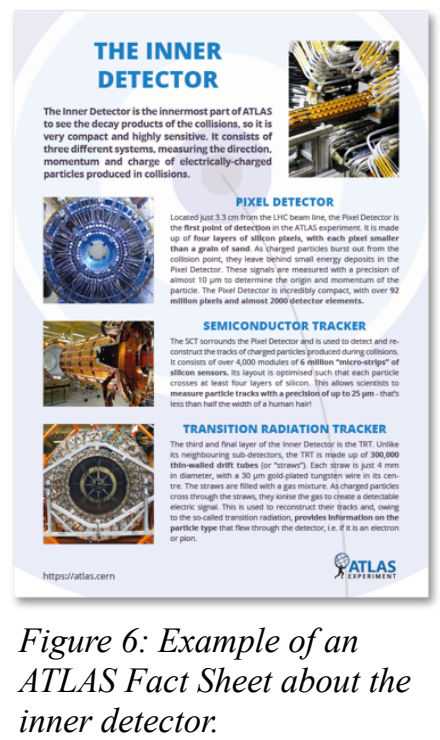

\subsection{The ATLAS Activity Sheets}

The ATLAS Activity Sheets [9] were developed based on the ATLAS colouring books and with the aim to allow the target audience (kids between 6 and 16 years old) to put into practice what they learned with the other ATLAS educational printables. Some of these sheets are available with different levels of difficulty and introduce some new concepts (for example, particle decays or antiparticles) but use the same particles used in the colouring books. For now, there are five types of activity sheets available in five languages.

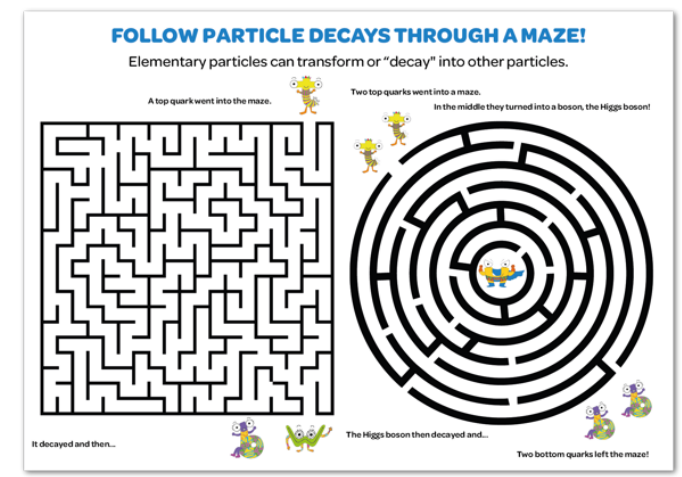

Figure 7: Example of an ATLAS Activity Sheet introducing the concept of particle decay.

\section{Summary}

The ATLAS experiment educational printables have been a success with the whole set being downloaded more than 140,000 times already. This data suggests significant interest in these materials and that people look for them to get more information about the ATLAS experiment. The ATLAS experiment aims to keep developing more of these resources and currently more educational printables are under development. 


\section{References}

[1] T. Ruão, et al., Science and strategic communication: how can universities attract high school students?, CECS, Braga 2015

[2] The Large Hadron Collider: https://home.cern/science/accelerators/largehadron-collider.

[3] ATLAS Collaboration, The ATLAS Experiment at the CERN Large Hadron Collider, 1361 JINST 3 (2008) S08003.

[4] The ATLAS Colouring Book: https://atlas.cern/resources/colouringbooks.

[5] Particles of the Universe: https://atlas.cern/resources/colouring-books.

[6] ATLAS Cheat Sheets: https: / /atlas.cern/resources/cheat-sheets.

[7] ATLAS public website: https://atlas.cern/.

[8] ATLAS Fact Sheets: https: //atlas.cern/resources/fact-sheets.

[9] ATLAS Activity Sheets: https: //atlas.cern/resources/colouring-books. 